\title{
El tratamiento precoz e intensivo con estatinas sería beneficioso durante los sindromes coronarios agudos
}

Early and intensive statin treatment appears to be beneficious during acute coronary syndromes

Hulten E, Arch Intern Med 2006;166:1814-21

Objetivo

Determinar si la administración precoz e intensiva de estatinas reduce los eventos cardiovasculares en pacientes con síndromes coronarios agudos (SCA).

\section{Fuente y selección de datos}

Ensayos controlados aleatorizados (ECA) desde 1974 a 2006 indizados en PubMed, MEDLINE, EMBASE, BIOSIS, SciSearch, PASCAL e Internacional Pharmaceutical Abstracts y Registro Cochrane de Ensayos Controlados. Debían comparar contra algún control en adultos el tratamiento precoz (dentro de los 14 días de internación) e intensivo (dosis mayores que las recomendadas en las guías de tratamiento ambulatorio) con estatinas. Los resultados considerados fueron los puntos finales combinados de cada ensayo y sus componentes individuales: muerte, infarto agudo de miocardio (IAM) y readmisión por isquemia.

\section{Resultados principales}

Fueron incluidos 17.963 pacientes de 13 estudios, cuatro de ellos multicéntricos e internacionales y con una mediana de 6 en el puntaje de Jadad *. Nueve estudios compararon estatinas con placebo y fueron evaluadas las siguientes estrategias: atorvastatina $80 \mathrm{mg}$, atorvastatina $20 \mathrm{mg}$, simvastatina $80 \mathrm{mg}$, pravastatina $40 \mathrm{mg}$ y fluvastatina $80 \mathrm{mg}$. La mediana de inicio del tratamiento fue cuatro días. A los seis meses se evidenció una reducción estadística- mente significativa en la incidencia de eventos cardiovasculares combinados, extendiéndose el beneficio durante 24 meses. Ver tabla 1. La reducción del LDL fue significativamente mayor en los pacientes tratados precozmente con estatinas a altas dosis y no hubo diferencias en la incidencia de los eventos adversos.

Tabla 1. Resultado primario compuesto y por componentes

\begin{tabular}{l|c|c|c|c}
\multirow{2}{*}{ Mes } & \multicolumn{4}{|l|}{ Hazard Ratio (IG95\%) } \\
\cline { 2 - 6 } & $\begin{array}{c}\text { Evento } \\
\text { cardilovascular }\end{array}$ & $\begin{array}{c}\text { Infarto de } \\
\text { miocardio }\end{array}$ & Ilsquemia & $\begin{array}{c}\text { Muerte } \\
\text { cardiovaseular }\end{array}$ \\
\hline $\mathbf{4}$ & $0,84(0,72-1,02)$ & $0,90(0,56-1,44)$ & $0,92(0,49-1,70)^{*}$ & $0,82(0,49-1,36)$ \\
\hline $\mathbf{6}$ & $0,76(0,70-0,84)^{*}$ & $0,53(0,22-1,26)$ & $0,50(0,44-0,58)^{*}$ & $0,69(0,07-6,80)$ \\
\hline $\mathbf{1 2}$ & $0,80(0,76-0,84)^{*}$ & $1,79(1,08-2,96)$ & $0,52(0,12-2,31)$ & $0,64(0,24-1,72)$ \\
\hline $\mathbf{2 4}$ & $0,81(0,77-0,87)^{*}$ & $0,43(0,24-0,78)^{*}$ & $0,70(0,51-0,97)^{*}$ & $0,74(0,63-0,86)^{*}$ \\
\hline Global & $0,84(0,76-0,94)^{*}$ & $0,89(0,60-1,33)$ & $0,68(0,50-0,92)^{*}$ & $0,76(0,66-0,87)^{*}$ \\
\hline
\end{tabular}

*Reducción estadísticamente significativa

\section{Conclusiones}

La administración de estatinas en altas dosis mejoraría a partir del cuarto mes de tratamiento la evolución clínica de los pacientes internados por SCA.

Palabras claves: estatinas, enfermedad cardiovascular, síndrome coronario agudo. Key words: statins, cardiovascular disease, acute coronary syndrome. Fuente de financiamiento: no reportada.

\section{Comentario}

Numerosos estudios han provisto evidencia sólida sobre la reducción asociada al tratamiento con estatinas de la morbimortalidad alejada de los pacientes con antecedentes de enfermedad coronaria. En base a estos datos, luego de un SCA el tratamiento con estatinas es una recomendación fuerte ${ }^{2,3}$

Según el paradigma actual, durante el SCA existe una placa accidentada que persistiría inestable durante varias semanas hipotéticamente, la administración precoz de altas dosis de estatinas contribuiría a una estabilización más rápida y completa de la placa responsable del evento índice, lo que estaría ligado al descenso plasmático de los lípidos o a los efectos pleiotrópicos de las estatinas ${ }^{4}$. Para demostrar este concepto es importante que el beneficio terapéutico se verifique precozmente (en semanas o meses) ya que de lo contrario, el efecto sería el mismo demostrado repetidamente en estudios previos de prevención secundaria, en los que la disminución del riesgo de eventos se inicia a partir de los 12 meses.

Si bien no hay evidencia de que haya existido sesgo de publicación, los estudios incluidos son muy heterogéneos* como para estar seguros del beneficio en cualquier circunstancia. Cuatro de ellos (PROVE-IT, A to Z, MIRACL y LIPID) representan el $94 \%$ de los pacientes y de estos cuatro, solo dos (MIRACL ${ }^{5}$ y PROVE- IT ${ }^{6}$ ) muestran efecto precoz. A su vez, el efecto precoz en MIRACL ocurre solo en un punto final blando (re-isquemia). En definitiva, el único estudio grande con efecto precoz sobre eventos duros es PROVE-IT

\section{Conclusión del comentador}

Aunque la hipótesis parece atractiva, no está todavía bien demostrada7. La comunidad médica tampoco parece convencida, ya que en la mayor parte de los centros se practica una estrategia "intermedia" consistente en la utilización de estatinas precozmente pero a dosis menores que las de estos estudios. Una razón adicional para la administración precoz reside en que condicionaría una mejor adherencia en estos pacientes, que casi siempre tienen indicación de prevención secundaria con estatinas.

Oscar Bazzino [ . Servicio de Cardiología. Hospital Italiano de Buenos Aires. ]

Bazzino O. Beneficio del tratamiento precoz e intensivo con estatinas durante los síndromes coronarios agudos. Evid. actual. práct. ambul; 10(1):8, enefeb.2007. Hulten E, Jackson JL, Douglas K, George S, Villines TC. The effect of early, intensive statin therapy on acute coronary syndrome: a meta-analysis of randomized controlled trials. Arch Intern Med. 2006; 166(17):1814-21. PMID: 17000936. Abstract en:

Referencias

1. Jadad AR, Moore RA, Carroll D, et al. Assessing the quality of reports of randomized clinical trials: is blinding necessary? Control Clin Trials. 1996; 17:1-12. 2. Expert Panel on Detection, Evaluation, and Treatment of High Blood Cholesterol in Adults. Executive Summary of the Third Report of the National Cholestero Education Program (NCEP) Expert Panel on Detection. Evaluation, and Treatment of High Blood Cholesterol in Adults (Adult Treatment Panel III). JAMA. 2001; 285:2486-97.

3. Smith SC, Jr., Blair SN, Bonow RO, Brass LM, Cerqueira MD, Dracup K, et al. AHA/ACC Guidelines for Preventing Heart Attack and Death in Patients With Atherosclerotic Cardiovascular Disease: 2001 Update: A Statement for Healthcare Professionals From the American Heart Association and the American College of Cardiology. Circulation 2001;104(13):1577-9.

4. Davignon J. Beneficial cardiovascular pleiotropic effects of statins. Circulation. 2004;109;(23)(suppl 1):III39-III43.

5. Gregory G. Schwartz, MD,PhD; Anders G. Olsson, MD,PhD; Michael D. Ezekowitz, MD,PhD; Peter Ganz, MD; Michael F. Oliver, MD; David Waters, MD; Andreas Zeiher, MD; Bernard R. Chaitman, MD; Sally Leslie, PhD; Theresa Stern, PhD; for the Myocardial Ischemia Reduction with Aggressive Cholesterol Lowering (MIRACL) Study Investigators. JAMA. 2001;285:1711-1718.

6. Ray KK, Cannon CP, McCabe CH, Cairns R, Tonkin AM, Sacks FM, Jackson G

7. Braunwald E; PROVE IT-TIMI 22 Investigators. J Am Coll Cardiol. 2005 Oct 18;46(8):1405-10. Early and late benefits of high-dose atorvastatin in patients with acute coronary syndromes: results from the PROVE IT-TIMI 22 trial.

8. Briel M, Schwartz GG, Thompson PL, et al. Effects of early treatment with statins on short-term clinical outcomes in acute coronary syndromes: a meta-analysis of randomized controlled trials. JAMA. 2006; 295:2046-56. 


\section{El uso del "chupete" durante el sueño se asoció a un menor riesgo de muerte súbita del lactante}

Objetivo
Examinar la asociación entre el uso del chupete durante el sueño y
el riesgo de muerte súbita del lactante (SMSL).

Diseño

Estudio caso-control* poblacional.

Lugar

Diez condados en California, EE.UU.

\section{Pacientes}

Para los casos se seleccionaron 185 madres de hijos con diagnóstico confirmado de SMSL. En cuanto a los controles un total de 312 madres de niños participaron. Se utilizó información de los certificados de nacimiento para identificar niños elegibles como controles del mismo condado en el que habían vivido los infantes muertos, apareados por raza materna.

\section{Evaluación de los factores de riesgo}

Todas las madres fueron interrogadas acerca del "índice del sueño", que para las madres de los niños muertos fue definida como la última noche y para las madres de los niños controles fue la noche anterior a la entrevista.

\section{Medición de resultados}

El resultado principal medido fue el uso del chupete durante el sueño. Se realizó además un análisis ajustado por potenciales confundidores. *

\section{Resultados Principales}

El Odds Ratio* ajustado para SMSL en niños que usaban chupete durante el último sueño fue de 0,08 ( $95 \%$ IC 0,03-0,21) es decir que su uso se asoció a una reducción de riesgo de muerte súbita del lactante, aún luego del ajuste por otros factores de riesgo conocidos (Ver Tabla 1). La reducción de riesgo asociada al uso del chupete parecería ser más importante ante condiciones adversas en el ámbito del sueño (posición prona o de costado, colecho con madre fumadora), sin embargo las diferencias observadas no fueron estadísticamente significativas.

Tabla 1. Uso de chupete y muerte súbita infantil.

\begin{tabular}{l|c|c|c|}
\hline \multicolumn{1}{c|}{ Resultado } & Gasos (n=169) & Gontroles(n=309) & $\begin{array}{c}\text { of ajustado* } \\
\text { (IE } 95 \%)\end{array}$ \\
\hline $\begin{array}{l}\text { Uso de chupete } \\
\text { la noche previa }\end{array}$ & $7(4.1 \%)$ & $73(23.6 \%)$ & $0,08(0,03$ a 0,21$)$ \\
\hline
\end{tabular}

* Por raza, edad y educación materna, tabaquismo durante el embarazo, edad del lactante, última posición para dormir, región y caracteristicas del parto.

\section{Conclusiones}

El uso del chupete durante el sueño parecería reducir el riesgo de muerte súbita y posiblemente la influencia de conocidos factores de riesgo en el ámbito del sueño.

Fuente de financiamiento: National Institute of Child Health and Human Development (NICHD) y National Institute on Deafness and Other Communication Disorders (NIDCD), National Institutes of Health.

\section{Comentario}

EI SMSL constituye una de las cuatro causas de mortalidad postneonatal más importantes. La tasa de muerte súbita en Argentina, que venía descendiendo en años anteriores, volvió a subir en el año 2002 a 0,53 por cada 1.000 nacidos vivos, aparentemente como consecuencia de la crisis económica. Los factores que se creen pueden relacionarse a esta mayor incidencia son el aumento de la pobreza, la disminución en el uso de la lactancia materna, la mayor incidencia de tabaquismo en los progenitores y el menor acceso a la educación preventiva'. La reducción en la incidencia de la muerte súbita luego de la campaña "back to sleep" en Estados Unidos mostró que el ambiente del sueño influencia fuertemente el riesgo de SMSL. En este estudio de casos y controles*, diseño ideal para evaluar un evento muy poco frecuente como la muerte súbita, el uso del chupete se asoció con una reducción de más de un $90 \%$ en el riesgo de SMSL, independientemente de otros factores de riesgo conocidos.

Las desventajas de este trabajo son que no es posible determinar un efecto de tipo dosis-respuesta, ya que se dificulta medir la intensidad del uso del chupete requerida para lograr la reducción del riesgo. Además el mismo diseño de casos y controles conlleva potenciales sesgos como el de selección de controles adecuados para comparar los casos y de recuerdo diferencial de los casos en relación a los controles que pueden comprometer la validez de la asociación detectada.

Otro punto a considerar es que se los resultados acerca de la magnitud de la protección son muy precisos, al observar la amplitud de los intervalos de confianza, por lo que no podemos saber con exactitud la magnitud de esta protección.

\section{Conclusiones del comentador}

Los resultados proveen cierta evidencia de que el uso del chupete podría reducir la incidencia del SMSL asociado o no a otros factores de riesgo. El uso del mismo puede ser una estrategia efectiva de salud pública si se recomendara entre los dos meses y el año de vida. Aunque antes de aconsejar en forma masiva esta estrategia preventiva desearíamos contar con datos provenientes de ensayos clínicos, probablemente debamos conformarnos con información de estudios como éste, debido a la baja incidencia de muerte súbita del lactante. Podemos, en todo caso, no desaconsejarlo.

Gonzalo Yamauchi Quintian [Unidad de Medicina Familiar y Preventiva del Hospital Italiano de Buenos Aires. ]

Yamauchi Quintian G. El uso del "chupete" durante el sueño se asoció a un menor riesgo de muerte súbita del lactante. Evid. Actual. Práct. Ambul. 9(6); 186 Nov-Dic. 2006. Comentado de: Use of a dummy (pacifier) during sleep and risk of sudden infant death syndrome (SIDS): population based case-control study. De-Kun Li, Marian Willinger, Diana B Petitti, y col. BMJ 2006; 332; 18-22. PMID: 16339767.

Referencias

1. Persico L y col. Síndrome de muerte súbita del lactante. Rev Fed Arg Cardiol 2004; 33: 341-344 